\title{
Joint optimal relay location and power allocation for ultra-wideband-based wireless body area networks
}

\author{
Jie Ding ${ }^{1 * \dagger}$, Eryk Dutkiewicz $^{1 \dagger}$ and Xiaojing Huang ${ }^{2 \dagger}$
}

\begin{abstract}
In this paper, we study the joint optimal relay location and power allocation problem for single-relay-assisted ultra-wideband (UWB)-based wireless body area networks (WBANs). Specifically, to optimize spectral efficiency (SE) for single-relay cooperative communication in UWB-based WBANs, we seek the relay with the optimal location together with the corresponding optimal power allocation. With proposed relay-location-based network models, the SE maximization problems are mathematically formulated by considering three practical scenarios, namely, along-torso scenario, around-torso scenario, and in-body scenario. Taking into account realistic power considerations for each scenario, the optimal relay location and power allocation are jointly derived and analyzed. Numerical results show the necessity of utilization of relay node for the spectral and energy-efficient transmission in UWB-based WBANs and demonstrate the effectiveness of the proposed scheme in particular for the around-torso and in-body scenarios. With the joint optimal relay location and power allocation, the proposed scheme is able to prolong the network lifetime and extend the transmission range in WBANs significantly compared to direct transmission.
\end{abstract}

Keywords: Body area networks; Spectral efficiency; Optimal relay location; Implant sensor node

\section{Introduction}

With the decreasing size and increasing capability of electronic devices, the wireless body area network (WBAN) is an enabling technology for pervasive healthcare by using several small and portable sensors on/in the human body $[1,2]$. For WBANs, the IEEE 802.15.6 standard has specified impulse radio ultra-wideband (IR-UWB) as its physical layer technology, owing to its simple electronics, high data rate capacity, and low power consumption, which is less likely to affect human tissues and cause interference to other medical equipments [3].

In healthcare applications, WBANs may be employed to monitor the vital signs of a patient, where spectral efficiency (SE) and reliability of emergency signal transmission are vitally important for the patient's life. However, the achievable SE of direct transmission in UWB-based

*Correspondence: jie.ding1@mq.edu.au

† Equal contributors

1 Department of Engineering, Macquarie University, Balaclava Road, North Ryde, NSW, 2109, Australia

Full list of author information is available at the end of the article
WBANs is often unsatisfactory because of the propagation blockage from the body torso as well as the limited transmit power due to the UWB regulatory limitations. Thus, SE is one of the most critical concerns in WBANs [4]. On the other hand, relay-assisted cooperative transmission has drawn considerable attention in wireless networks which can improve the information rate and link reliability effectively [5].

Considerable studies have been conducted in wireless sensor networks (WSNs) regarding the SE of the relayassisted communications [6-8]. In [6], power allocation between a source and relay was optimized to maximize the SE in single-relay-based cooperative networks. In [7], joint relay selection and power allocation strategy for multiuser amplify-and-forward (AF) networks were studied to maximize users' SEs. In [8], power allocation and relay selection schemes were proposed to achieve the maximum SE and minimum outage probability for multiple relay-assisted cooperative networks. These studies reveal that cooperative transmission is an effective way that can greatly increase the SE in WSNs.

\section{是 Springer}

(c) 2015 Ding et al: licensee Springer. This is an Open Access article distributed under the terms of the Creative Commons Attribution License (http://creativecommons.org/licenses/by/4.0), which permits unrestricted use, distribution, and reproduction in any medium, provided the original work is properly credited. 
Unlike WSNs, UWB-based WBANs have some unique properties such as analog transmission, distinct channel characteristics, and limited network size, where the signal strength in a WBAN is mostly affected by the physical location of the nodes in relation to each other as well as the human body [9]. As a result, the aforementioned existing schemes and results on the SE in WSNs may be inadequate if they are applied to UWB-based WBANs directly. This fact motivated us to study the SE of relay-assisted cooperative transmission in UWB-based WBANs. For cooperative transmission, some related studies have been conducted in WBANs [10-16]. Particularly, channel modeling and system diversity were analyzed in [10]. In [11], the energy efficiency of cooperative transmission was investigated with constrained outage probability. Cooperative scheduling schemes were proposed to decrease inter-BAN interference and increase packet reception rate of intra-BAN communications in [12]. In [13] and [14], packet error rate performance evaluation of two-hop links against the direct link was presented over narrow-band channels. In [15], the energy efficiency of cooperative transmission was considered from a relay selection perspective for UWB-based WBANs and the energy-efficient performance was analyzed with different relay regions. In [16], the reliability and energy efficiency of two-hop cooperative communication was assessed theoretically in terms of outage probability and bit error rate for narrow-band medical services. Although these studies have demonstrated that cooperative communication can be effectively implemented in WBANs, the SE of cooperative communication is still an open issue for UWB-based WBANs. Furthermore, to the best of our knowledge, research on the SE of cooperative communication related to the optimal relay location (RL) and power allocation (PA) in UWB-based WBANs has been rarely conducted, especially for scenarios involving both 'onbody to on-body'and 'in-body to on-body' propagation links.

To this end, this work aims to solve the joint optimal RL and PA problem to optimize the SE in single-relay-assisted UWB-based WBANs. Three practical transmission scenarios are investigated herein, which are along-torso scenario, around-torso scenario, and in-body scenario, respectively. Each scenario refers to a specific physical location between source and destination nodes in relation to each other. For each scenario, we seek the relay with the optimal location to achieve the maximum SE, together with the corresponding optimal power allocation. More precisely, generic relay-location-based network models are proposed for UWB-based WBANs firstly. Taking into account realistic power considerations for each scenario, the SE optimization problem is then mathematically formulated and the optimal RL and PA are jointly derived to achieve the maximum SE. The analysis on the optimal RL and PA is given accordingly. Numerical results show the necessity of utilization of relay node for the spectral and energy-efficient transmission in UWB-based WBANs and reveal that the relay location is an influential parameter in WBANs.

By utilizing an on-body relay node with the joint optimal relay location and power allocation, the transmission range in WBANs can be extended effectively and the power consumption can be transferred from the sensor node to the relay node, in which the lifetime of the sensor node can be prolonged significantly, particularly for the around-torso and in-body scenarios.

\section{System and channel models}

\section{System scenarios}

In this paper, we consider a basic WBAN which is composed of three types of nodes: one wearable or implant sensor node, one body network coordinator, and one relay node. The sensor node is used to monitor the physiological states of a person periodically, e.g., measuring heartbeat or recording body activities, and it is connected to the coordinator directly or through the relay node. Normally, the coordinator is a personal digital assistant (PDA) attached on the human body. Note that the application type of the sensor node and its location in a WBAN depend on the requirement of patient.

In this work, the single-relay-based cooperative transmission is considered in UWB-based WBANs. We herein study a standard two-phase AF cooperative protocol. It consists of two time slots with equal duration, in which the sensor node $S$ (source) broadcasts its signal to the coordinator $D$ (destination) and the relay node $R$ during the first time slot, and in the second time slot, $R$ forwards its received signal to $D$. For cooperative transmission in WBANs, three typical scenarios are investigated, namely, along-torso scenario, around-torso scenario, and in-body scenario. The along-torso scenario refers to the condition that $S$ and $D$ are on the same side of the human body. The around-torso scenario refers to the condition that $S$ and $D$ are on the different sides of the human body, and the in-body scenario refers to the condition that $S$ is placed inside the human body.

\section{Proposed cooperative models}

To evaluate the impact of relay location on the SE and find out the optimal relay location for each considered scenario, two relay-location-based cooperative models are developed for on-body transmissions and in-body transmissions, respectively.

In Figure 1, the around-torso scenario is considered. In this scenario, we only study the case that all nodes are only located on the front side of the human body or on the back side of the human body rather than the limbs. Thus, 


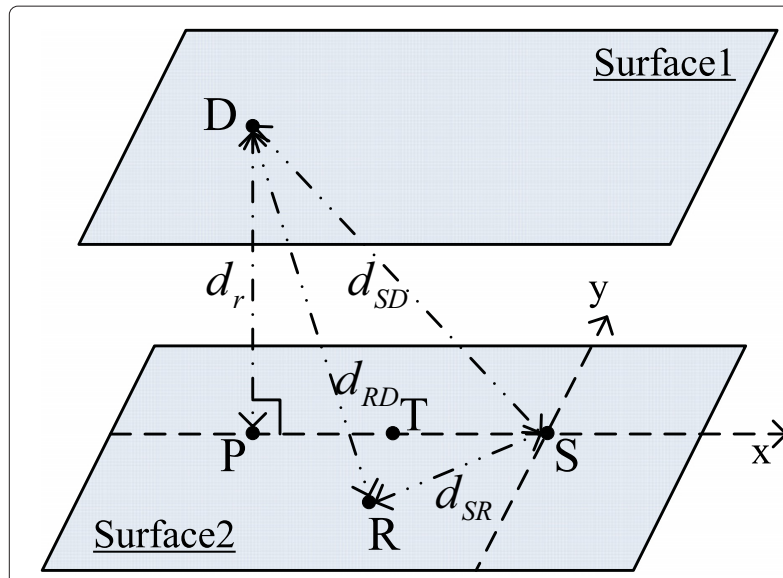

Figure 1 Proposed cooperative model for on-body transmissions in WBANs.

a two-surface system model can be established under the reasonable assumption that each side of the human body is approximately treated as a level surface, as shown in Figure 1. Without loss of generality, we assume that $D$ is located on the front side of the human body (Surface1) and $S$ and $R$ are located on the back side of the human body (Surface2). The distance between these two surfaces is $d_{r}\left(d_{r} \geq 0\right) . d_{S D}, d_{S R}$, and $d_{R D}$ denote the distances from $S$ to $D, S$ to $R$, and $R$ to $D$, respectively. We denote the projection of $D$ on Surface2 by point $P$, and point $T$ is located in the middle between $P$ and $S$. To represent the location of $R$, we construct a xy-plane on Surface2, where $S$ is set to be the origin point and the $\mathrm{x}$-axis is along $P$ to $S$. It is worth noting that this equivalent cooperative model will be simplified to the one for the alongtorso scenario when $d_{r}=0$. In this case, $D$ is located on the same surface with $S$. Thus, we can consider the along-torso scenario as a special case in the around-torso scenario.

In Figure 2, the in-body scenario is considered where $S$ is located inside the human body (e.g., chest) and $R$ and $D$ are located on the same side of the human body. The penetration depth from $S$ to the body surface is $d_{r}\left(d_{r}>0\right)$. Similarly to the on-body cooperative model, we denote the projection of $S$ on the body surface by point $P$. On the body surface, we can construct a xy-plane to present the location of $R$, where $P$ is set to be the origin point and the $\mathrm{x}$-axis is along $P$ to $D$.

Comparing the two proposed models, we can see that their structures are very similar.

Thus, all the three scenarios can be analytically similar for solving the joint optimal RL and PA problem. In the sequel, we focus on the SE optimization in the around-torso scenario and only detail the solution of the considered problem for the around-torso scenario ${ }^{\mathrm{a}}$.

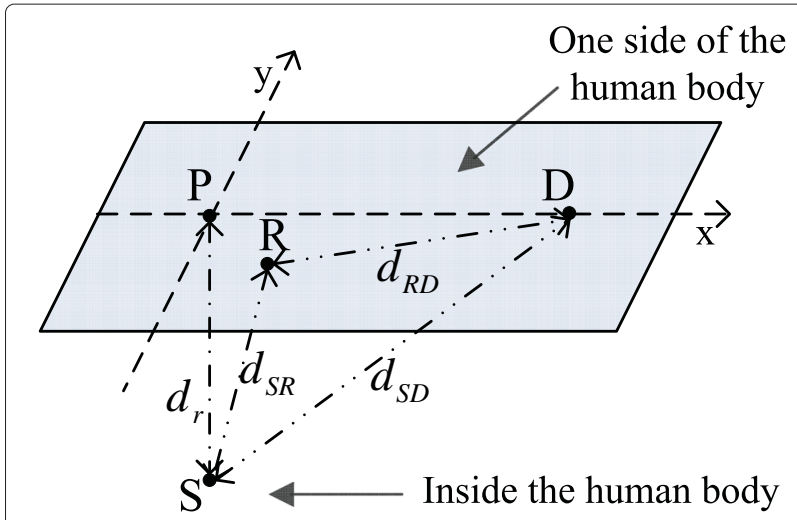

Figure 2 Proposed cooperative model for in-body transmissions in WBANs.

In the around-torso scenario, with a given coordinates $\left\{x_{r}, y_{r}\right\}$ for $R$, we have

$$
d_{S R}\left(x_{r}, y_{r}\right)=\sqrt{x_{r}^{2}+y_{r}^{2}}
$$

and

$$
d_{R D}\left(x_{r}, y_{r}\right)=\sqrt{\left(x_{r}+d_{S D} \sin \theta\right)^{2}+y_{r}^{2}+d_{r}^{2}},
$$

where $\theta=\arccos \left(d_{r} / d_{S D}\right)$.

\section{Channel models}

Accordingly, three types of channel models are considered in UWB-based WBANs for cooperative transmission, namely, along-torso channel model, around-torso channel model, and in-body channel model.

In this paper, the effect of the body motion and the scattering inside the human body is taken into consideration. Since we assume that all on-body nodes are only located on the front side of the human body or on the back side of the human body rather than the limbs, the impact of the body motion can be ignored in the alongtorso and in-body scenarios based on the observations in the existing literature $[17,18]$. However, a walking motion produces measurable fluctuations in the around-torso scenario, which cannot be ignored [18]. Therefore, the shadowing (variations) from the body motion, modeled as a Lognormal distribution, is included into the aroundtorso path loss model. On the other hand, the scattering inside the human body is caused by the different material dielectric properties along the propagation path, which is also necessary to be considered in the in-body channel model [19]. Table 1 summarizes the corresponding parameters for the path loss (including shadowing) models [18-20]. Notice that $H_{0}$ represents the shadowing from the body motion, which is a Gaussian distributed random variable with mean 0.27 and standard deviation 1.5 [18]. $H_{1}$ represents the scattering inside the human body, which 
Table 1 UWB-based WBAN path loss (including shadowing) models

\begin{tabular}{ll}
\hline Along-torso model & $P L_{0}^{\mathrm{dB}}(d)=L_{0}+10 n_{0} \log _{10}\left(\frac{d}{d_{0}}\right)$ \\
\hline$L_{0}[\mathrm{~dB}]$ & 44.6 \\
\hline$n_{0}$ & 3.1 \\
\hline$d_{0}[m]$ & 0.1 \\
\hline Around-torso model & $P L_{1}^{\mathrm{dB}}(d)=L_{1}+10 n_{1} \log _{10}\left(\frac{d}{d_{1}}\right)+H_{0}$ \\
\hline$L_{1}[\mathrm{~dB}]$ & 48.4 \\
\hline$n_{1}$ & 5.9 \\
\hline$d_{1}[m]$ & 0.1 \\
\hline In-body model & $P L_{2}^{\mathrm{dB}}(d)=L_{2}+a\left(d / d_{2}\right)^{n_{2}}+H_{1}$ \\
\hline$L_{2}[\mathrm{~dB}]$ & 10 \\
\hline$a$ & 0.987 \\
\hline$n_{2}$ & 0.85 \\
\hline$d_{2}[m]$ & 0.001 \\
\hline$L_{i}$ isthepat lossatte
\end{tabular}

$L_{i}$ is the path loss at the reference distance, for $i=0,1,2 . d_{i}$ is the reference distance, for $i=0,1,2 . n_{i}$ is the path loss exponent, for $i=0,1,2 . a$ is a fitting constant. $H_{i}$ is Gaussian distributed random variable, for $i=0,1$.

is a Gaussian distributed random variable with zero mean and standard deviation 7.84 [19].

Since signals are transmitted over the along-torso channel for the $S$ - $R$ link and over the around-torso channels for the other two links, both channel models are used in the around-torso scenario ${ }^{b}$.

From the path loss models defined in the log scale, the path losses in the linear scale from $S$ to $D, S$ to $R$, and $R$ to $D$ in the around-torso scenario can be obtained as,

$$
\begin{aligned}
& P L_{S D}\left(d_{S D}\right)=10^{P L_{1}^{d B}\left(d_{S D}\right) / 10}=M_{1} d_{S D}^{n_{1}}, \\
& P L_{S R}\left(d_{S R}\right)=10^{P L_{0}^{d B}\left(d_{S R}\right) / 10}=M_{0} d_{S R}^{n_{0}},
\end{aligned}
$$

and

$$
P L_{R D}\left(d_{R D}\right)=10^{P L_{1}^{d B}\left(d_{R D}\right) / 10}=M_{1} d_{R D}^{n_{1}},
$$

respectively. $M_{0}=\left(1 / d_{0}\right)^{n_{0}} 10^{L_{0} / 10}$ and $M_{1}=\left(1 / d_{1}\right)^{n_{1}}$ $10^{L_{1} / 10} 10^{H_{0} / 10}$ are constant.

For all the links considered, the energy-normalized channel impulse response (CIR) in the around-torso scenario can be written as

$$
h_{k}(t)=\sum_{l=0}^{L_{k}-1} \alpha_{l, k} \delta\left(t-\tau_{l, k}\right)
$$

where $k \in\{S D, S R, R D\}$ denotes the links from $S$ to $D, S$ to $R$, and $R$ to $D$, respectively. $L_{k}$ is the number of multipaths, $\tau_{l, k}$ is the delay of the $l$ th path, and $\alpha_{l, k}$ is the gain of the $l$ th path. Since real signals are employed in UWB systems, each path gain is real also. Further detail on the delay profile for along-torso and around-torso links can be found in $[20]^{\mathrm{c}}$.
In the next section, the SE optimization in the aroundtorso scenario is investigated, which is taken as an example for the other two scenarios. We first introduce the SE of direct transmission. Then, the optimal SE of cooperative transmission is formulated based on the proposed model, and the joint optimal RL and PA is derived accordingly.

\section{Spectral efficiency optimization}

\section{SE of direct transmission}

Without loss of generality, we present the IR-UWB signal transmission with pulse-amplitude modulation (PAM).

When data is modulated, pulse shaped, and transmitted repeatedly over $N_{f}$ consecutive frames at $S$, the received signal at $D$ is given by

$$
r_{S D}(t)=b \sqrt{\frac{P_{S}}{P L_{S D}\left(d_{S D}\right)}} \sum_{j=0}^{N_{f}-1} g_{S D}\left(t-j T_{f}\right)+n_{S D}(t), \quad b= \pm 1,
$$

where $b$ is the transmitted symbol and $P_{s}$ is the transmit power of $S$ and the value of $P_{S}$ depends on the battery power limit of $S$. In on-body transmissions, we assume that $P_{s} \leq P_{\max }^{o}$, where $P_{\max }^{o}$ is the maximum transmit power for the on-body node, which is constrained by the Federal Communications Commission (FCC) power spectral density (PSD) emission limit for UWB signals (accordingly, we assume that $P_{s} \leq P_{\max }^{i}$ in the in-body scenario).

$$
g_{S D}(t)=\omega(t) * h_{S D}(t)=\sum_{l=0}^{L_{S D}-1} \alpha_{l, S D} \omega\left(t-\tau_{l, S D}\right),
$$

where $*$ represents convolution. $\omega(t)$ denotes the ultrashort pulse waveform with $T_{w}$ duration, which has the unit energy $\int_{t=0}^{T_{f}} \omega^{2}(t) d t=1 . T_{f}$ is the duration of frame, and it is set to be large enough to avoid the inter-symbol interference (ISI). $n_{S D}(t)$ is the additive white Gaussian noise (AWGN) with zero mean and variance $\sigma_{n}^{2}$.

At $D$, a received pulse waveform matched filter is employed. After summing up all the outputs over $N_{f}$ frames, the decision statistic $b$ at $D$ can be written as

$$
b_{S D}=b N_{f} \sqrt{\frac{P_{s}}{P L_{S D}\left(d_{S D}\right)}} \bar{\xi}_{S D}+\hat{n}_{S D},
$$

where $\bar{\xi}_{S D}$ is the captured multipath energy during $T_{f}$ at $D$ in direct transmission and $\hat{n}_{S D}$ is a white Gaussian noise with zero mean and variance $N_{f} \bar{\xi}_{S D} \sigma_{n}^{2}$.

With (6), SE (unit:bits/s/Hz) for direct transmission can be given by

$$
C_{S D}=\frac{1}{N_{f}} \log _{2}\left(1+\gamma_{S D}\right)
$$


where $\gamma_{S D}=\frac{N_{f} P_{s} \bar{\xi}_{S D}}{P L_{S D}\left(d_{S D}\right) \sigma_{n}^{2}}$ is the received signal to noise ratio (SNR).

\section{Formulation for the SE optimization in cooperative transmission}

Similar to $D, R$ is also equipped with a matched filter. With the considered AF cooperative protocol, the SE of the single-relay cooperative transmission can be given by

$$
C_{S R D}=\frac{1}{2 N_{f}} \log _{2}\left(1+\gamma_{1}+\gamma_{2}\right),
$$

where $\gamma_{1}$ is the received SNR at $D$ in the first time slot and

$$
\gamma_{2}=\frac{\gamma_{S R} \gamma_{R D}}{\gamma_{S R}+\gamma_{R D}+1} \approx \frac{\gamma_{S R} \gamma_{R D}}{\gamma_{S R}+\gamma_{R D}}
$$

is the received SNR at $D$ in the second time slot. $\gamma_{S R}$ and $\gamma_{R D}$ are the received SNRs for the links $S-R$ and $R-D$, respectively.

In the around-torso scenario, the expressions of $\gamma_{1}, \gamma_{S R}$, and $\gamma_{R D}$ are given by

$$
\begin{aligned}
& \gamma_{1}=\frac{N_{f} P_{1} \xi_{S D}}{P L_{S D}\left(d_{S D}\right) \sigma_{n}^{2}}, \\
& \gamma_{S R}=\frac{N_{f} P_{1} \xi_{S R}}{P L_{S R}\left(d_{S R}\right) \sigma_{n}^{2}}, \\
& \gamma_{R D}=\frac{N_{f} P_{2} \xi_{R D}}{P L_{R D}\left(d_{R D}\right) \sigma_{n}^{2}},
\end{aligned}
$$

where $P_{1}$ and $P_{2}$ are the transmit power at $S$ and $R$, respectively. In this paper, to make a fair comparison with direct transmission, we assume that the total transmit power resource in cooperative transmission is not more than that in direct transmission, i.e., $P_{1}+P_{2} \leq P_{S} . \xi_{S D}$, $\xi_{S R}$, and $\xi_{R D}$ are the captured multipath energy during $T_{f}$ for the links $S-D, S-R$, and $R-D$ in cooperative transmission, respectively.

Substituting (1), (2), and (9) to (12) into (8), we have

$$
\begin{aligned}
C_{S R D}\left(x_{r}, y_{r}, P_{1}, P_{2}\right) & =\frac{1}{2 N_{f}} \log _{2}(1+\underbrace{\frac{N_{f} P_{1} \xi_{S D}}{P L_{S D}\left(d_{S D}\right) \sigma_{n}^{2}}}_{\gamma_{1}\left(P_{1}\right)} \\
& +\underbrace{\frac{1}{P_{L_{S R}\left(d_{S R}\left(x_{r}, y_{r}\right)\right) \sigma_{n}^{2}}^{N_{f} P_{1} \xi_{S R}}+\frac{P L_{R D}\left(d_{R D}\left(x_{r}, y_{r}\right) \sigma_{n}^{2}\right.}{N_{f} P_{2} \xi_{R D}}}}_{\gamma_{2}\left(x_{r}, y_{r}, P_{1}, P_{2}\right)}) .
\end{aligned}
$$

Obviously, $C_{S R D}$ is the function of variables $\left\{x_{r}, y_{r}, P_{1}, P_{2}\right\}$. To obtain the maximum $C_{S R D}$ in the around-torso scenario, we must find the optimal set $\left\{x_{r}^{o}, y_{r}^{o}, P_{1}^{o}, P_{2}^{o}\right\}$ that makes $C_{S R D}\left(x_{r}, y_{r}, P_{1}, P_{2}\right)$ maximized. Thus, the joint optimal RL and PA problem for the
SE optimization in the around-torso scenario can be mathematically formulated as

$$
\begin{array}{cl}
\underset{x_{r}, y_{r}, P_{1}, P_{2}}{\operatorname{maximize}} & C_{S R D}\left(x_{r}, y_{r}, P_{1}, P_{2}\right) \\
\text { subject to } & \mathrm{C} 1:\left(x_{r}+\frac{d_{S D} \sin \theta}{2}\right)^{2}+y_{r}^{2} \leq\left(\frac{d_{S D} \sin \theta}{2}\right)^{2}, \\
& \mathrm{C} 2: x_{r} \leq \delta, \\
& \mathrm{C} 3: P_{1}+P_{2} \leq P_{s},
\end{array}
$$

where $\mathrm{C} 1$ is imposed to guarantee that $R$ is only located in the circle centered at $T$ with radius $\frac{d_{S D} \sin \theta}{2}$. This special circle for $R$ is considered based on the fact that we can always find a corresponding relay location within the circle which can provide a better performance than those beyond the circle. $\mathrm{C} 2$ is imposed to guarantee that $\left\{x_{r}=\right.$ $\left.0, y_{r}=0\right\}$ has to be beyond $\mathrm{C} 1$ since $R$ cannot coincide with $S$, where $|\delta|$ is a very small constant and we set $-10^{-6}<\delta<0$. With $C 1$ and $C 2$, we have $d_{S R}, d_{R D}<d_{S D}$.

As mentioned in the previous section, similar objective functions and constrains can be established for the other two scenarios. Notice that, in the in-body scenario, another constrain must be taken into consideration, i.e., C4: $P_{2} \leq P_{\max }^{o}$, which is imposed to guarantee that $P_{2}$ meets the requirement of the FCC PSD limit for the UWB signals since $R$ is on the body surface. In the next part, we detail the derivation and solution of the optimal RL and PA problem for the SE optimization in the around-torso scenario. For the other two scenarios, the related optimal RL and PA can also be achieved with similar derivations.

\section{Joint optimal RL and PA in cooperative transmission}

Since $\log _{2}(1+x)$ is a monotonically increasing function of $x$, we can rewrite the optimization problem as

$$
\begin{array}{ll}
\underset{x_{r}, y_{r}, P_{1}, P_{2}}{\operatorname{maximize}} & \gamma_{1}\left(P_{1}\right)+\gamma_{2}\left(x_{r}, y_{r}, P_{1}, P_{2}\right) \\
\text { subject to } & \mathrm{C} 1, \mathrm{C} 2, \mathrm{C} 3 .
\end{array}
$$

From (10), we can see that $\gamma_{1}\left(P_{1}\right)$ is a linear function of $P_{1}$, which indicates that $\gamma_{1}\left(P_{1}\right)$ is concave. To prove that $\gamma_{2}\left(x_{r}, y_{r}, P_{1}, P_{2}\right)$ is a concave function, we introduce the following proposition and theorem.

Proposition 1. $\frac{P L_{S R}\left(d_{S R}\left(x_{r}, y_{r}\right)\right) \sigma_{n}^{2}}{N_{f} P_{1} \xi_{S R}}$ and $\frac{P L_{R D}\left(d_{R D}\left(x_{r}, y_{r}\right)\right) \sigma_{n}^{2}}{N_{f} P_{2} \xi_{R D}}$ are both convex for $P_{1}, P_{2}>0$.

Proof. Please refer to Appendices.

Theorem 1. Given that $f(x)$ and $g(y)$ are both convex, $\phi(x, y)=f(x)+g(y)$ is convex.

Proof. Please refer to Appendices. 
By Proposition 1 and Theorem $1,1 / \gamma_{2}\left(x_{r}, y_{r}, P_{1}, P_{2}\right)$ is a convex function and $1 / \gamma_{2}\left(x_{r}, y_{r}, P_{1}, P_{2}\right)>0$. Hence, $\gamma_{2}\left(x_{r}, y_{r}, P_{1}, P_{2}\right)$ is concave w.r.t. C1-C3. As a result, the objective function in (15) is concave.

Thus, it is concluded that the joint RL and PA problem for the around-torso scenario is a nonlinear convex optimization problem, which can be solved by using the Lagrange multiplier method with Karush-Kuhn-Tucker (KKT) conditions [21].

The Lagrangian of (15) can be given by

$$
\begin{aligned}
& \mathcal{L}\left(x_{r}, y_{r}, P_{1}, P_{2}, \mu_{1}, \mu_{2}, \mu_{3}\right) \\
& =\gamma_{1}\left(P_{1}\right)+\gamma_{2}\left(x_{r}, y_{r}, P_{1}, P_{2}\right)-\mu_{1} \\
& \quad \times\left(\left(x_{r}+\frac{d_{S D} \sin \theta}{2}\right)^{2}+y_{r}^{2}-\left(\frac{d_{S D} \sin \theta}{2}\right)^{2}\right) \\
& \quad-\mu_{2}\left(x_{r}-\delta\right)-\mu_{3}\left(P_{1}+P_{2}-P_{s}\right),
\end{aligned}
$$

where $\mu_{1}, \mu_{2}, \mu_{3} \geq 0$ are the Lagrange multipliers connected to $\mathrm{C} 1-\mathrm{C} 3$. Taking the stationarity condition of each variable, we can prove that $\mu_{1}=\mu_{2}=0$ and the solution $\left\{x_{r}^{o}, y_{r}^{o}, P_{1}^{o}, P_{2}^{o}\right\}$ satisfies (17) to (20):

$$
\begin{aligned}
& y_{r}^{o}=0, \\
& \frac{x_{r}^{o} n_{0} M_{0} P_{2}^{o} \xi_{R D}}{n_{1} M_{1} P_{1}^{o} \xi_{S R}} A_{1}\left(x_{r}^{o}, 0\right)+\left(x_{r}^{o}+d_{S D} \sin \theta\right) \\
& \frac{\xi_{S D}}{M_{1} d_{S D}^{n_{1}}}+\frac{\xi_{S R} \xi_{R D}\left(P_{2}^{o}\right)^{2} A_{3}\left(x_{r}^{o}, 0\right)}{\left(P_{2}^{o} A_{3}\left(x_{r}^{o}, 0\right)+P_{1}^{o} A_{4}\left(x_{r}^{o}, 0\right)\right)^{2}} \\
& =\frac{\xi_{S R} \xi_{R D}\left(P_{1}^{o}\right)^{2} A_{4}\left(x_{r}^{o}, 0\right)}{\left(P_{2}^{o} A_{3}\left(x_{r}^{o}, 0\right)+P_{1}^{o} A_{4}\left(x_{r}^{o}, 0\right)\right)^{2}},
\end{aligned}
$$$$
\frac{x_{r}^{o} n_{0} M_{0} P_{2}^{o} \xi_{R D}}{n_{1} M_{1} P_{1}^{o} \xi_{S R}} A_{1}\left(x_{r}^{o}, 0\right)+\left(x_{r}^{o}+d_{S D} \sin \theta\right) A_{2}\left(x_{r}^{o}, 0\right)=0,
$$

and

$$
P_{1}^{o}+P_{2}^{o}=P_{s}
$$

where $A_{1}\left(x_{r}, y_{r}\right)=\left(x_{r}^{2}+y_{r}^{2}\right)^{\frac{n_{0}-2}{2}}$ and $A_{2}\left(x_{r}, y_{r}\right)=$ $\left(\left(x_{r}+d_{S D} \sin \theta\right)^{2}+y_{r}^{2}+d_{r}^{2}\right)^{\frac{n_{1}-2}{2}} \cdot A_{3}\left(x_{r}, y_{r}\right)=\xi_{R D} M_{0}$ $\left(x_{r}^{2}+y_{r}^{2}\right)^{\frac{n_{0}}{2}}$ and $A_{4}\left(x_{r}, y_{r}\right)=\xi_{S R} M_{1}\left(\left(x_{r}+d_{S D} \sin \theta\right)^{2}+y_{r}^{2}+\right.$ $\left.d_{r}^{2}\right)^{\frac{n_{1}}{2}}$.

Based on (17) to (20), the optimal solution $\left\{x_{r}^{o}, y_{r}^{o}, P_{1}^{o}, P_{2}^{o}\right\}$ is achieved for the $\mathrm{SE}$ maximization problem in the around-torso scenario. For the other two scenarios, similar proofs and derivations are not repeated herein.

\section{Discussions:}

(1) A typical application for the around-torso scenario in a WBAN is the post-neck surgery tracking for patient, where a sensor node is placed on the neck of patient to measure the angular motion of the neck and send the updated status of recovery to the coordinator in the front pocket.

(2) In a WBAN, the number of nodes and their application types depend on the requirement of patient. Based on the 802.15.6 standard [3], a multiple access mode known as 'improvised access' is supported in the WBAN standard, whereby the coordinator can inform nodes that they have been granted one-off exclusive time slots to transmit or receive information. Thus, in a particular time slot, only one node is active and other nodes are inactive (in a sleep mode or act as relays). Without loss of generality, an ideal medium access control (MAC) layer is assumed in this paper.

(3) The proposed joint optimal RL and PA scheme aims to provide an insight into the design of healthcare applications with respect to the proper placement of the wearable relay node along with the optimal transmit power level in WBANs. When the number of nodes in a WBAN is small (e.g., only one or two nodes are attached on or in the human body), relays can be added to the WBAN with the optimal placements based on the proposed scheme, which would not cause the comfort issue to the patient. When the node density in a WBAN is high, it is inappropriate to add additional relays for nodes. In fact, inactive nodes can be selected as relays to cooperate in forwarding the data from the active node towards the coordinator. Thus, the proposed scheme can be also considered as a source of inspiration for the relay selection in this case. For instance, the coordinator can adopt the proposed scheme for the current active node. Based on the information about the corresponding optimal relay location, the inactive node, whose location is closest to the optimum, can be selected as a relay candidate.

\section{Simulation}

To evaluate the SE of the proposed single-relay-assisted transmission scheme in UWB-based WBANs, numerical simulations are conducted in this section, which consist of two parts: the SE evaluation for on-body transmissions and the SE evaluation for in-body transmissions. The simulations are performed by MATLAB with Monte Carlo method. MATLAB's optimization toolbox is used for solving the convex programming in the simulations. All the simulation results are averaged over 5,000 channel realizations.

In simulations, $T_{w}$ and $T_{f}$ are chosen to be 2 and $150 \mathrm{~ns}$, respectively. $N_{f}$ is set to be 4 , the noise power density is set to be $-174 \mathrm{dBm} / \mathrm{Hz}$, and the system bandwidth $B$ is $50 \mathrm{MHz}$ [3]. Since the average FCC PSD emission limit for on-body UWB transmissions is $-41.3 \mathrm{dBm} / \mathrm{MHz}$ [22], the maximum average transmit power $P_{\text {ave }}$ is $-14.3 \mathrm{dBm}$. With the duty cycle $T_{w} / T_{f}, P_{\max }^{o}=P_{\text {ave }} * T_{f} / T_{w}=$ $4 \mathrm{dBm}$ [23]. For the in-body transmissions, we set $P_{\max }^{i} \leq$ $10 \mathrm{dBm}$ considering the emission limit and safety inside the human body [23]. According to the scale of the human 
body, $d_{S D}$ is very limited in UWB-based WBANs. Without loss of generality, we consider the case that $0.5 \mathrm{~m}$ $\leq d_{S D} \leq 0.8 \mathrm{~m}$ for on-body transmissions and $0.2 \mathrm{~m}$ $\leq d_{S D} \leq 0.3 \mathrm{~m}$ for in-body transmissions. At $R$ and $D$, we assume that all of the dispersive energies are captured without considering the ISI.

\section{SE evaluation for on-body transmissions}

In this part, the SE performance for on-body transmissions is evaluated. We first analyze the features of the optimal RL and PA in each scenario. Then, the comparison between the proposed scheme and other transmission schemes are presented.

\section{Analysis of the optimal $R L$ and $P A$}

Table 2 presents the averaged joint optimal set $\left\{\mathrm{E}\left\{x_{r}^{o}\right\}, \mathrm{E}\left\{y_{r}^{o}\right\}, \mathrm{E}\left\{P_{1}^{o}\right\}, \mathrm{E}\left\{P_{2}^{o}\right\}\right\}$ with various values of $d_{S D}$ for the along-torso scenario and the around-torso scenario, respectively. It is shown that the simulation results match the theoretical derivation in 'Spectral efficiency optimization' section. The optimal relay is always located on the negative $\mathrm{x}$-axis for both scenarios, and we can see that the averaged optimal relay location is very close to the point $P$ in the around-torso scenario. With $P_{1}^{o}+P_{2}^{o}=P_{s}$, it is evident that the optimal SE-based scheme always uses the maximum power for capacity maximization. For the along-torso scenario, we can see that the optimal power allocation ratio $\frac{P_{1}^{o}}{P_{2}^{o}}$ is invariant with different

Table 2 Averaged optimal set $\left\{\mathrm{E}\left\{x_{r}^{o}\right\}, \mathrm{E}\left\{y_{r}^{o}\right\}, \mathrm{E}\left\{P_{1}^{o}\right\}, \mathrm{E}\left\{P_{2}^{o}\right\}\right\}$ for on-body transmissions

\begin{tabular}{|c|c|c|c|c|}
\hline \multicolumn{5}{|c|}{ Along-torso scenario $\left(d_{r}=0\right)$} \\
\hline$d_{S D}$ & $0.5 \mathrm{~m}$ & $0.6 \mathrm{~m}$ & $0.7 \mathrm{~m}$ & $0.8 \mathrm{~m}$ \\
\hline $\mathrm{E}\left\{x_{r}^{\circ}\right\}$ & -0.30 & -0.36 & -0.42 & -0.48 \\
\hline $\mathrm{E}\left\{y_{r}^{\circ}\right\}$ & 0 & 0 & 0 & 0 \\
\hline $\mathrm{E}\left\{P_{1}^{o}\right\}$ & $0.70 P_{s}$ & $0.70 P_{s}$ & $0.70 P_{s}$ & $0.70 P_{s}$ \\
\hline $\mathrm{E}\left\{P_{2}^{\circ}\right\}$ & $0.30 P_{s}$ & $0.30 P_{s}$ & $0.30 P_{s}$ & $0.30 P_{s}$ \\
\hline \multicolumn{5}{|c|}{ Around-torso scenario when $d_{r}=0.2 \mathrm{~m}$} \\
\hline$d_{S D}$ & $0.5 \mathrm{~m}$ & $0.6 \mathrm{~m}$ & $0.7 \mathrm{~m}$ & $0.8 \mathrm{~m}$ \\
\hline $\mathrm{E}\left\{x_{r}^{\circ}\right\}$ & -0.41 & -0.51 & -0.61 & -0.70 \\
\hline $\mathrm{E}\left\{y_{r}^{O}\right\}$ & 0 & 0 & 0 & 0 \\
\hline $\mathrm{E}\left\{P_{1}^{O}\right\}$ & $0.40 P_{s}$ & $0.48 P_{s}$ & $0.54 P_{s}$ & $0.59 P_{s}$ \\
\hline$\underline{E}\left\{P_{2}^{o}\right\}$ & $0.60 P_{s}$ & $0.52 P_{s}$ & $0.46 P_{s}$ & $0.41 P_{s}$ \\
\hline \multicolumn{5}{|c|}{ Around-torso scenario without body motion when $d_{r}=0.2 \mathrm{~m}$} \\
\hline$d_{S D}$ & $0.5 \mathrm{~m}$ & $0.6 \mathrm{~m}$ & $0.7 \mathrm{~m}$ & $0.8 \mathrm{~m}$ \\
\hline $\mathrm{E}\left\{x_{r}^{O}\right\}$ & -0.42 & -0.52 & -0.62 & -0.72 \\
\hline $\mathrm{E}\left\{\mathrm{y}_{r}^{\circ}\right\}$ & 0 & 0 & 0 & 0 \\
\hline $\mathrm{E}\left\{P_{1}^{O}\right\}$ & $0.42 P_{s}$ & $0.50 P_{s}$ & $0.56 P_{s}$ & $0.61 P_{s}$ \\
\hline $\mathrm{E}\left\{P_{2}^{\circ}\right\}$ & $0.58 P_{s}$ & $0.50 P_{s}$ & $0.44 P_{s}$ & $0.39 P_{s}$ \\
\hline
\end{tabular}

$d_{S D}$. This is due to the fact that, since in the along-torso scenario, all signals are transmitted over the along-torso channels; the optimal relay location relative to $S$ and $D$ is unchanged when $d_{S D}$ varies. Thus, $\frac{P_{1}^{o}}{P_{2}^{o}}$ does not vary. As opposed to the along-torso scenario, $\frac{P_{1}^{o}}{P_{2}^{o}}$ varies with $d_{S D}$ in the around-torso scenario. This can be explained by the fact that signals are transmitted over the along-torso channel only for the $S-R$ link and over the around-torso channels for the other two links. The change of $d_{S D}$ has an impact on the optimal relay location relative to $S$ and $D$ and therefore $\frac{P_{1}^{o}}{P_{2}^{o}}$. Moreover, with a fixed $d_{r}$ and larger $d_{S D}$, it can be observed that more power is allocated to $S$ to make sure that the maximum SE can be achieved in the around-torso scenario.

In addition, we also show the $\left\{\mathrm{E}\left\{x_{r}^{o}\right\}, \mathrm{E}\left\{y_{r}^{o}\right\}, \mathrm{E}\left\{P_{1}^{o}\right\}, \mathrm{E}\left\{P_{2}^{o}\right\}\right\}$ in the around-torso without considering the body motion, where we simply discard the body motion variable $H_{0}$ from the around-torso channel model. Comparing the values with and without the body motion, we can clearly see that the proposed scheme is able to adjust the optimal RL and PA readily when external circumstances vary. To overcome the shadow fading, the optimal RL in the proposed scheme moves towards point $S$ slightly and accordingly more power is allocated to $R$. In this manner, $\gamma_{S R}$ and $\gamma_{R D}$ are balanced adaptably under the condition that the body motion is present, and thus the SE is optimized in this circumstance.

In the following, the SE performance of the proposed joint optimal scheme is exhibited for each scenario. We will show that the proposed scheme is capable of overcoming the effect of the shadowing and achieving a SE performance close to that without considering the shadowing. Moreover, with the features of the optimal RL and PA presented in Table 2, some intended comparisons are also made in the around-torso scenario to further verify the effectiveness of our proposed scheme.

\section{SE performance comparison}

In Figure 3, the average SE is compared between direct transmission and the proposed scheme in the along-torso scenario $\left(d_{r}=0\right)$. As shown in this figure, direct transmission is much more spectral efficient than the proposed scheme and the performance gap between the two schemes increases significantly as $P_{S}$ increases. This is because when a line-of-sight (LoS) between $S$ and $D$ is present in UWB-based WBANs, the path loss exponent is small and a high SNR can be achieved by direct transmission. However, the pre-log factor $\frac{1}{2}$ in (8) causes a substantial loss for cooperative transmission in the SE in this case and this loss is especially more significant in the higher SNR regime. Thus, we can see that the proposed scheme is not beneficial to the spectral efficiency in the along-torso scenario. In other words, when the source and 


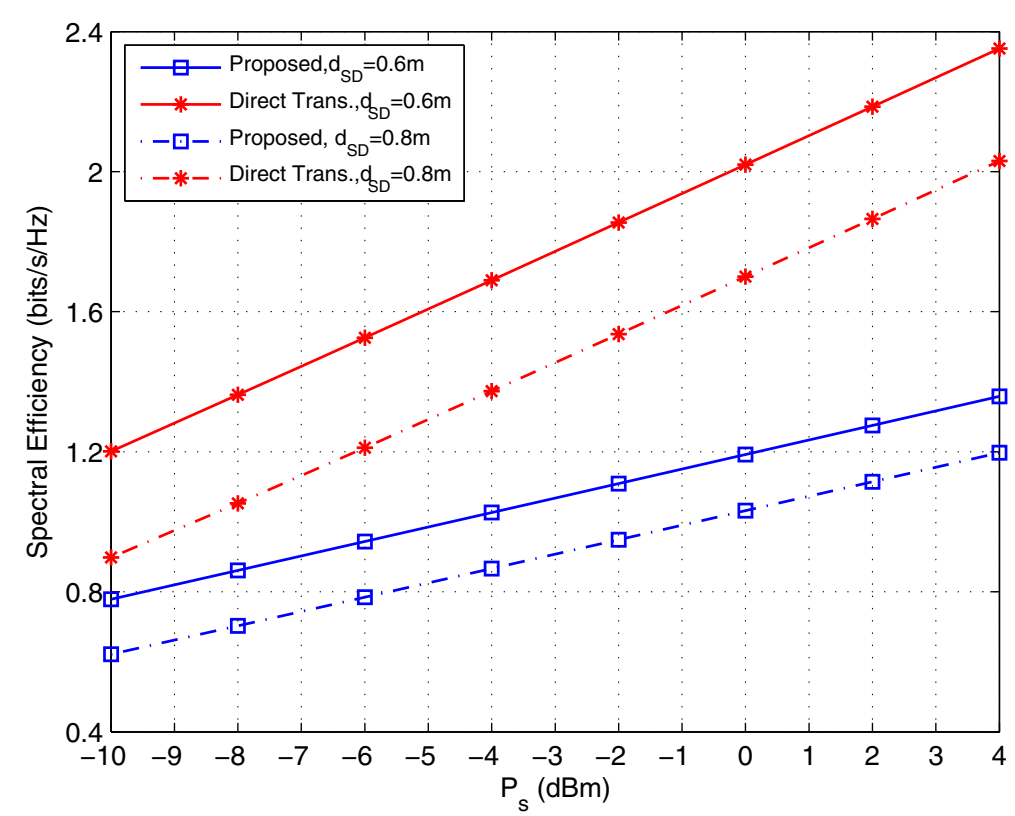

Figure 3 Average $\mathrm{SE}$ versus $P_{s}$ for the along-torso scenario.

destination are on the same side of the human body, the source prefers to transmit its signals to the destination directly.

Figure 4 depicts the average SE versus $P_{S}$ with $d_{S D}=0.6$ $\mathrm{m}$ and $d_{r}=0.2 \mathrm{~m}$ in the around-torso scenario. To evaluate the performance of the proposed scheme, comparisons are made with: 1) the proposed scheme without considering the shadowing due to the body motion, 2) the optimal
PA scheme at point $P, 3)$ the optimal PA scheme at point $T, 4)$ selection amplify-and-forward (S-AF) scheme [8], and 5) direct transmission. In the optimal PA schemes at points $P$ and $T$, the optimal $P_{1}$ and $P_{2}$ are exploited to maximize the SE when $R$ is fixed at points $P$ and $T$, respectively. In the S-AF scheme, we assume that six relays are randomly located in the circle defined in $\mathrm{C} 1$ and each relay has the same power as $S$, i.e., $\frac{P_{s}}{2}$. The relay that

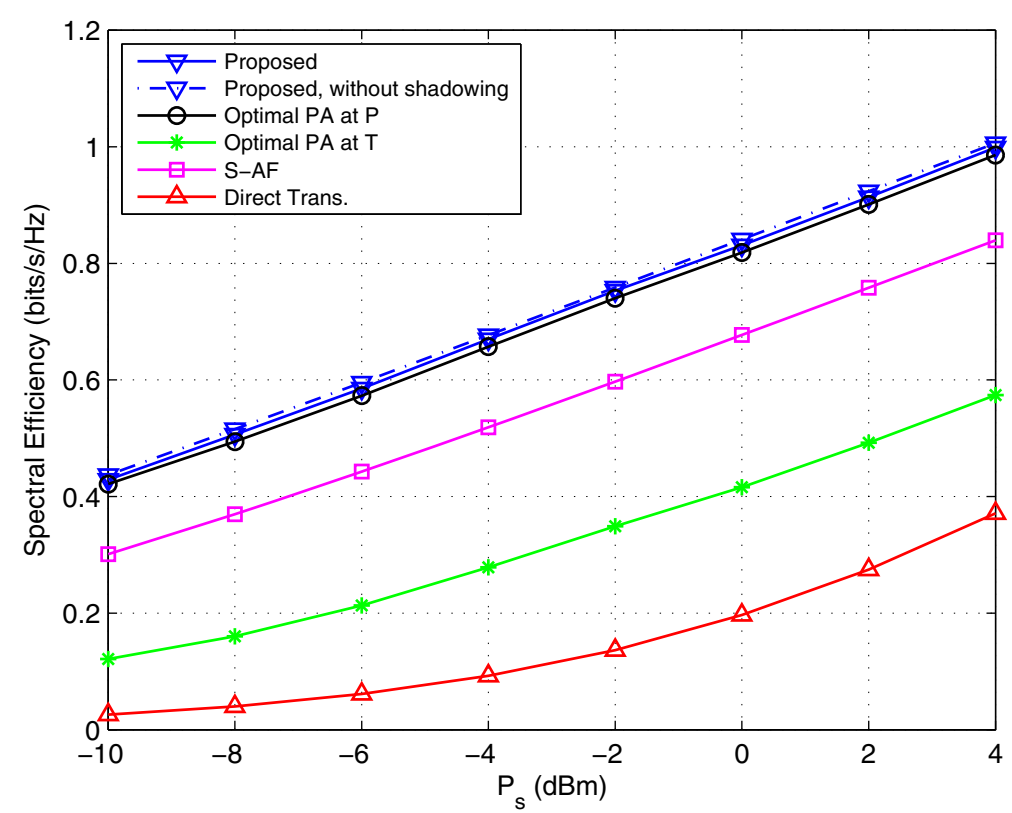

Figure 4 Average SE versus $P_{S}$ in the around-torso scenario when $d_{S D}=0.6 \mathrm{~m}$ and $d_{r}=0.2 \mathrm{~m}$. 
can achieve the maximum SE is selected. Undoubtedly, the case without considering the body motion is ideal since the shadow fading is neglected and thus the optimal SE in this case can be treated as an up-bound that can be achieved by the proposed scheme. Evidently, the proposed scheme can achieve a close to up-bound performance when the shadowing is considered, which means that the proposed scheme is capable of overcoming the effect of the shadowing by adjusting its optimal RL and PA adaptably, as shown in Table 2. It is also observed that the proposed scheme can achieve the best SE among all the schemes when shadowing is taken into account and all the cooperative transmission schemes outperform direct transmission. Moreover, the SE with the optimal PA at $P$ is very close to the optimum in the proposed scheme, which indicates that the location of $P$ is a good choice to place the relay node in this case. Compared to direct transmission, we can see that the proposed scheme can provide a remarkable performance improvement and up to 17 times improvement can be achieved when the battery of the sensor node is very limited (e.g., $P_{s} \leq-8 \mathrm{dBm}$ ). From the perspective of power consumption, we notice that the transmit power at $S$ by using the proposed scheme can be much less (more than $14 \mathrm{~dB}$ ) compared to that by direct transmission when the same SE is achieved. This evidence indicates that the lifetime of the sensor node can be prolonged considerably with the assistance of the relay, which demonstrates that the proposed scheme is an effective way to enhance the SE and prolong the lifetime of the sensor node.

To further explore the performance of the proposed scheme in the around-torso scenario, the average SE versus $d_{S D}$ with different $d_{r}$ and fixed $P_{s}=4 \mathrm{dBm}$ is illustrated in Figure 5. Comparisons are made with: 1) the optimal PA scheme at point $P, 2)$ the equal PA scheme at point $T, 3) \mathrm{S}$-AF scheme, and 4) direct transmission. Similarly to Figure 4, it is shown that the the proposed scheme is the most spectral efficient among all the schemes. We also notice that direct transmission is very sensitive to $d_{S D}$. That is to say, without the LoS between $S$ and $D$, the significant propagation loss in the aroundtorso scenario would affect the performance of direct transmission adversely. By contrast, the proposed scheme exhibits a weak dependence upon $d_{S D}$, which reveals that the proposed scheme can extend the transmission range effectively in the around-torso scenario.

\section{SE evaluation for in-body transmissions}

For in-body transmissions, we evaluate the SE performance in the same way as on-body transmissions.

\section{Analysis of the optimal $R L$ and $P A$}

Table 3 shows the averaged joint optimal set $\left\{\mathrm{E}\left\{x_{r}^{o}\right\}, \mathrm{E}\left\{y_{r}^{o}\right\}, \mathrm{E}\left\{P_{1}^{o}\right\}, \mathrm{E}\left\{P_{2}^{o}\right\}\right\}$ with various values of $P_{s}$ for

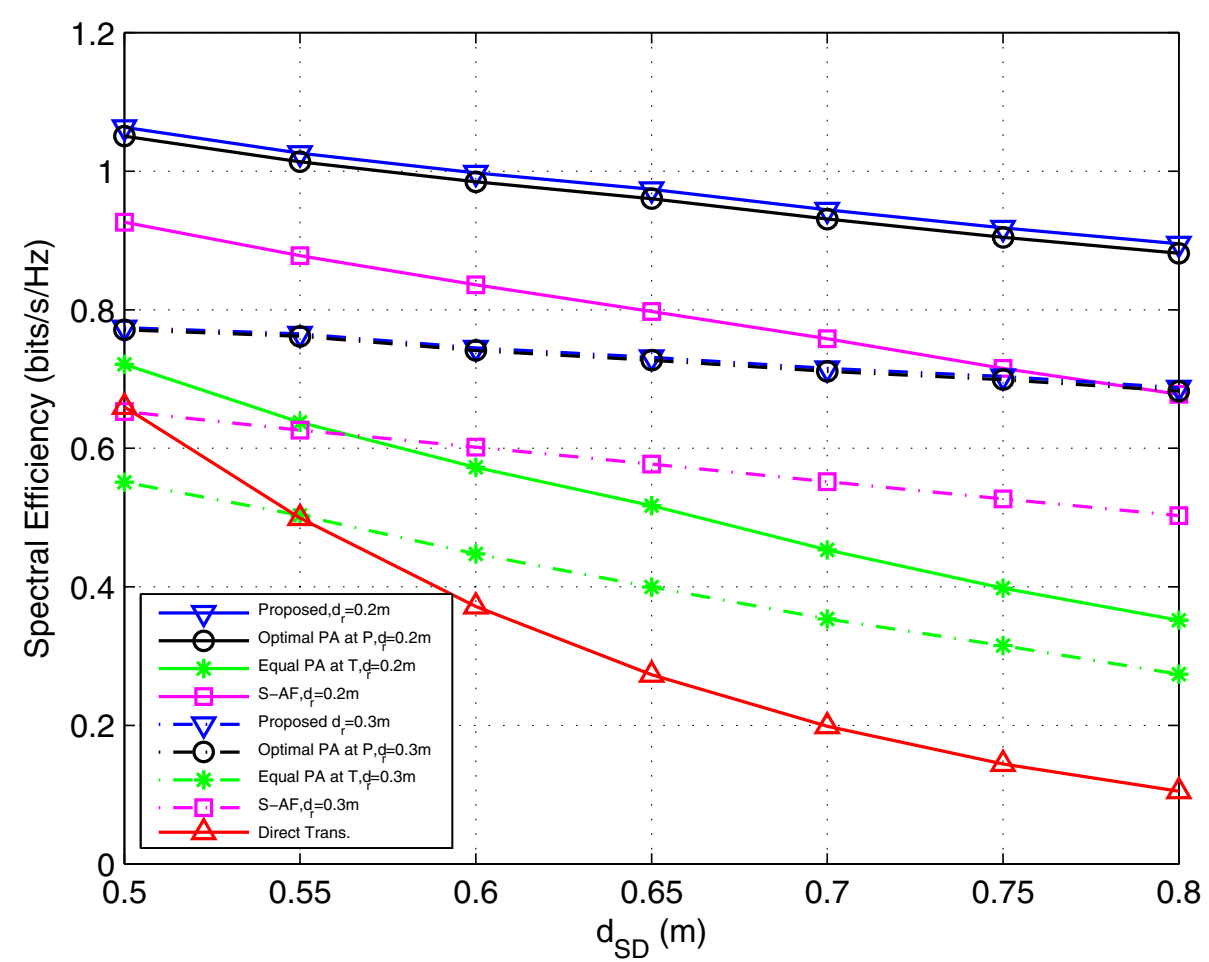

Figure 5 Average SE versus $d_{S D}$ in the around-torso scenario with different $d_{r}$ and $P_{S}=4 \mathrm{dBm}$. 
Table 3 Averaged optimal set $\left\{\mathrm{E}\left\{x_{r}^{o}\right\}, \mathrm{E}\left\{y_{r}^{o}\right\}, \mathrm{E}\left\{P_{1}^{o}\right\}, \mathrm{E}\left\{P_{2}^{o}\right\}\right\}$ for in-body transmissions

\begin{tabular}{|c|c|c|c|c|c|c|}
\hline \multicolumn{7}{|c|}{ In-body scenario with $d_{r}=5 \mathrm{~cm}$ and $d_{S D}=0.2 \mathrm{~m}$} \\
\hline $\mathrm{E}\left\{x_{r}^{0}\right\}$ & 0.033 & 0.033 & 0.033 & 0.033 & 0.038 & 0.055 \\
\hline$\left\{y_{r}^{0}\right\}$ & 0 & 0 & 0 & 0 & 0 & 0 \\
\hline$\left\{P_{1}^{\circ}\right\}$ & $0.28 P_{s}$ & $0.28 P_{s}$ & $0.28 P_{s}$ & $0.28 P_{s}$ & $P_{s}-P_{\max }^{O}$ & $P_{s}-P_{\max }^{O}$ \\
\hline$\left\{P_{2}^{\circ}\right\}$ & $0.72 P_{s}$ & $0.72 P_{s}$ & $0.72 P_{s}$ & $0.72 P_{s}$ & $P_{\max }^{0}$ & $P_{\max }^{o}$ \\
\hline \multicolumn{7}{|c|}{ In-body scenario with $d_{r}=5 \mathrm{~cm}$ and $d_{S D}=0.3 \mathrm{~m}$} \\
\hline$P_{s}$ & $-10 \mathrm{dBm}$ & $-6 \mathrm{dBm}$ & $-2 \mathrm{dBm}$ & $2 \mathrm{dBm}$ & $6 \mathrm{dBm}$ & $10 \mathrm{dBm}$ \\
\hline$E\left\{x_{r}^{o}\right\}$ & 0.038 & 0.038 & 0.038 & 0.038 & 0.050 & 0.67 \\
\hline $\mathrm{E}\left\{y_{r}^{0}\right\}$ & 0 & 0 & 0 & 0 & 0 & 0 \\
\hline$\left\{P_{1}^{o}\right\}$ & $0.18 P_{s}$ & $0.18 P_{s}$ & $0.18 P_{s}$ & $0.18 P_{s}$ & $P_{s}-P_{\max }^{O}$ & $P_{s}-P_{\text {max }}^{O}$ \\
\hline$E\left\{P_{2}^{O}\right\}$ & $0.82 P_{s}$ & $0.82 P_{s}$ & $0.82 P_{s}$ & $0.82 P_{s}$ & $P_{\max }^{O}$ & $P_{\max }^{O}$ \\
\hline
\end{tabular}

the in-body scenario. Since the on-body transmit power $P_{2}$ cannot excess the FCC PSD limit, i.e., $P_{\max }^{o}$, we can see that $P_{2}^{o}$ is limited to be equal to $P_{\max }^{o}$ in the high total transmit power regimes, i.e., $P_{s} \geq 6 \mathrm{dBm}$. This result indicates that the SE of the proposed scheme may increase gradually over the high total transmit power regimes since $P_{2}^{o}$ is constrained by the transmit power allowance $P_{\max }^{o}$ and $P_{2}^{o}$ plays a critical role on the SE performance considering the $R-D$ link transmission takes the advantage of experiencing much lower path loss compared to the $S-R$ link in the in-body scenario. Moreover, with the optimal
PA in the proposed scheme, it turns out that $S$ only need to transmit a small amount of $P_{s}$ in the low-to-moderate regimes so that the optimal SE can be achieved. This evidence reveals that the proposed scheme can transfer the most power consumption from the implant node to the on-body relay. In this way, the lifetime of the implant node can be prolonged, which is very beneficial to the implant node considering its battery cannot be easily replaced.

\section{SE performance comparison}

Figure 6 presents the average SE versus $P_{s}$ with different $d_{S D}$ and fixed $d_{r}=5 \mathrm{~cm}$ in the in-body scenario. As expected, we can see that the proposed scheme can provide a significant improvement in term of SE compared to direct transmission. Moreover, it can be noticed that the SE of the proposed scheme ascends in a gradual way and the SE with optimal PA at point $P$ approaches a constant over the high total transmit power regimes. This result verifies the accuracy of our analysis based on Table 3 well. In addition, it is observed that the SE of direct transmission deteriorates drastically when $d_{S D}=0.3 \mathrm{~m}$, which indicates that direct transmission cannot provide a satisfactory transmission performance in this scenario due to the severe propagation conditions within the human body. Thus, the employment of relay node is essential for in-body transmissions.

In conclusion, direct transmission may not be able to provide an acceptable transmission performance in

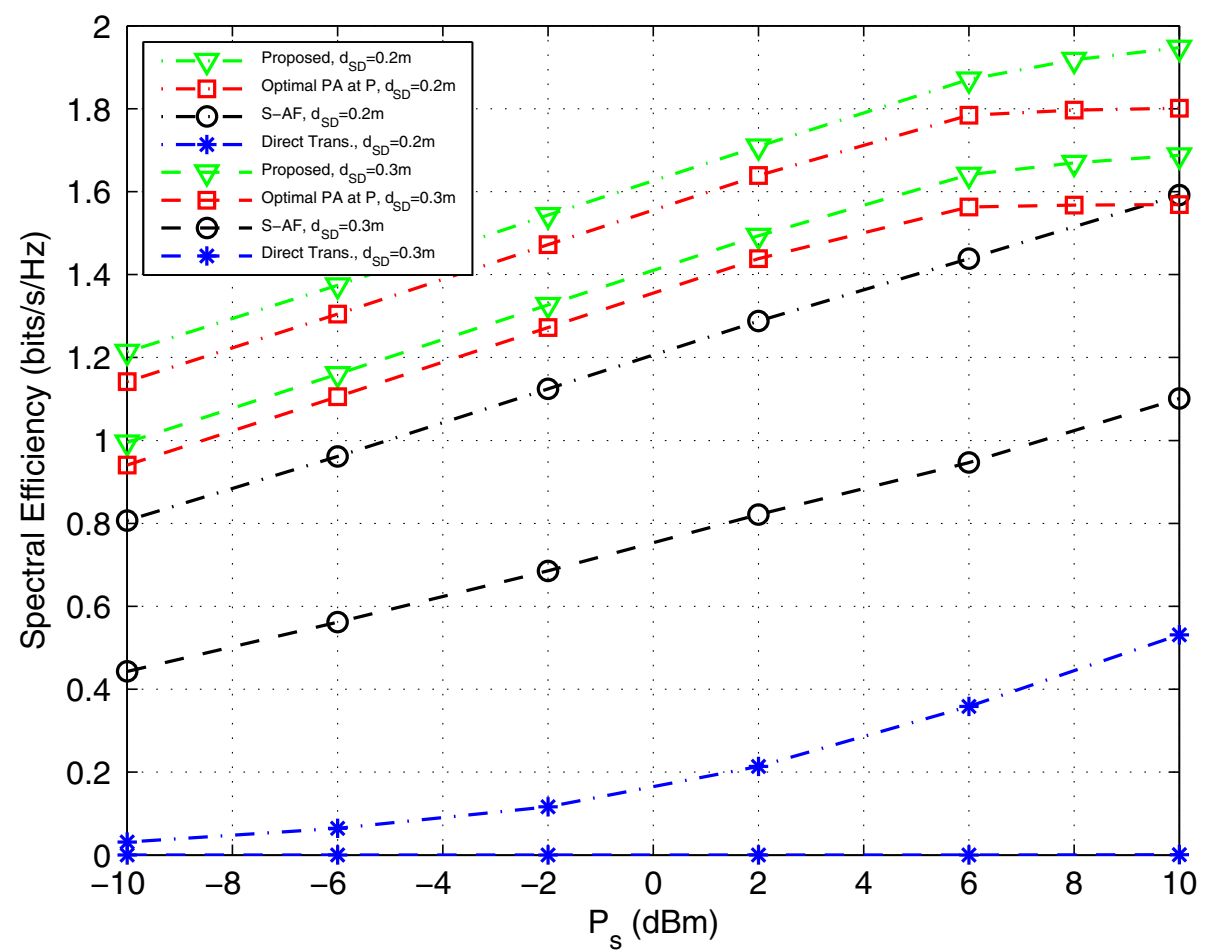

Figure 6 Average SE versus $P_{s}$ for the in-body scenario with different $d_{S D}$ and $d_{r}=5 \mathrm{~cm}$. 
UWB-based WBANs except in the along-torso scenario. In contrast, the proposed scheme is an effective approach for the spectral and energy-efficient transmission, particularly in the around-torso and in-body scenarios. By utilizing an on-body relay node with the joint optimal $\mathrm{RL}$ and PA, the transmission range in WBANs can be extended effectively and the power consumption can be transferred from the sensor node to the relay node, in which the lifetime of the sensor node can be prolonged significantly compared to direct transmission.

\section{Conclusions}

In this paper, healthcare communication scheme is considered for UWB-based WBANs, where single-relaybased cooperative transmission is investigated to enhance the SE performance for various practical communication scenarios regarding the physical location between source and destination nodes in relation to each other. The SE optimization problem is mathematically formulated, and the optimal relay location and power allocation are jointly exploited towards providing insight into the design of healthcare applications with respect to the proper location and required transmission power of the wearable relaying device. Due to the presence of the LoS in the along-torso scenario, we show that direct transmission is preferable to the cooperative transmission. However, by utilizing an on-body relay node with the joint optimal relay location and power allocation in the around-torso and in-body scenarios, it is exhibited that the transmission range in UWB-based WBANs can be extended effectively and the power consumption can be transferred from the sensor node to the relay node, in which the lifetime of the sensor node can be prolonged significantly compared to direct transmission.

\section{Endnotes}

${ }^{a}$ Apart form the different channel parameters and the location of nodes in relation to each other, no extra challenges emerge in the along-torso and in-body scenarios for solving the SE optimization problem.

${ }^{\mathrm{b}}$ Similarly, the along-torso and in-body channel models are used in the in-body scenario, and only the along-torso channel model is used in the along-torso scenario.

${ }^{\mathrm{c}}$ For the in-body link, the corresponding delay profile can be found in [19].

\section{Appendices}

\section{Proof of Proposition 1}

For notational simplicity, we define

$f_{1}\left(x_{r}, y_{r}, P_{1}\right)=\frac{P L_{S R}\left(d_{S R}\left(x_{r}, y_{r}\right)\right) \sigma_{n}^{2}}{N_{f} P_{1} \xi_{S R}}=\frac{\left(x_{r}^{2}+y_{r}^{2}\right)^{\frac{n_{0}}{2}} M_{0} \sigma_{n}^{2}}{N_{f} P_{1} \xi_{S R}}$ and

$$
\begin{aligned}
f_{2}\left(x_{r}, y_{r}, P_{2}\right) & =\frac{P L_{R D}\left(d_{R D}\left(x_{r}, y_{r}\right)\right) \sigma_{n}^{2}}{N_{f} P_{2} \xi_{R D}} \\
& =\frac{\left(\left(x_{r}+d_{S D} \sin \theta\right)^{2}+y_{r}^{2}+d_{r}^{2}\right)^{\frac{n_{1}}{2}} M_{1} \sigma_{n}^{2}}{N_{f} P_{2} \xi_{R D}} .
\end{aligned}
$$

Then, we denote $\mathbf{H}\left(f_{1}\left(x_{r}, y_{r}, P_{1}\right)\right)$ and $\mathbf{H}\left(f_{2}\left(x_{r}, y_{r}, P_{2}\right)\right)$ as the Hessian matrixes of functions $f_{1}\left(x_{r}, y_{r}, P_{1}\right)$ and $f_{2}\left(x_{r}, y_{r}, P_{2}\right)$, respectively. The determinants of $\mathbf{H}\left(f_{1}\left(x_{r}, y_{r}, P_{1}\right)\right)$ and $\mathbf{H}\left(f_{2}\left(x_{r}, y_{r}, P_{2}\right)\right)$ are given by

$$
\left|\mathbf{H}\left(f_{1}\left(x_{r}, y_{r}, P_{1}\right)\right)\right|=\frac{M_{0}^{3} \sigma_{n}^{6}\left(n_{0}^{3}-2 n_{0}^{2}\right)\left(x_{r}^{2}+y_{r}^{2}\right)^{\frac{3 n_{0}-4}{2}}}{P_{1}^{5} N_{f}^{3} \xi_{S R}^{3}},
$$

and

$$
\left|\mathbf{H}\left(f_{2}\left(x_{r}, y_{r}, P_{2}\right)\right)\right|=\frac{M_{1}^{3} \sigma_{n}^{6}}{N_{f}^{3} \xi_{R D}^{3}} \frac{\left(n_{1}^{3}-2 n_{1}^{2}\right)\left(\left(x_{r}+d_{S D} \sin \theta\right)^{2}+y_{r}^{2}\right)+2 n_{1}^{2} d_{r}^{2}}{P_{2}^{5}\left(\left(x_{r}+d_{S D} \sin \theta\right)^{2}+y_{r}^{2}+d_{r}^{2}\right)^{\frac{6-3 n_{1}}{2}}},
$$

respectively.

Since $n_{0}^{3}-2 n_{0}^{2}>0$ and $n_{1}^{3}-2 n_{1}^{2}>0, \mathbf{H}\left(f_{1}\left(x_{r}, y_{r}, P_{1}\right)\right)$ and $\mathbf{H}\left(f_{2}\left(x_{r}, y_{r}, P_{2}\right)\right)$ are both positive definite matrixes with $P_{1}, P_{2}>0$. In other words, $f_{1}\left(x_{r}, y_{r}, P_{1}\right)$ is jointly convex w.r.t. $x_{r}, y_{r}$, and $P_{1} . f_{2}\left(x_{r}, y_{r}, P_{2}\right)$ is jointly convex w.r.t. $x_{r}$, $y_{r}$, and $P_{2}$.

\section{Proof of Theorem 1}

Since $f(x)$ and $g(y)$ are convex, we have

$$
f\left(t x_{1}+(1-t) x_{2}\right) \leq t f\left(x_{1}\right)+(1-t) f\left(x_{2}\right),
$$

and

$$
g\left(t y_{1}+(1-t) y_{2}\right) \leq \operatorname{tg}\left(y_{1}\right)+(1-t) g\left(y_{2}\right),
$$

where $x_{1}$ and $x_{2}$ are any two points for function $f(x)$ and $y_{1}$ and $y_{2}$ are any two points for function $g(y) .0<t<1$. We define that $z_{1}=\left\{x_{1}, y_{1}\right\}$ and $z_{2}=\left\{x_{2}, y_{2}\right\}$. Then, we can write

$$
\begin{aligned}
\phi\left(t z_{1}+(1-t) z_{2}\right) & =\phi\left(t x_{1}+(1-t) x_{2}, t y_{1}+(1-t) y_{2}\right) \\
& =f\left(t x_{1}+(1-t) x_{2}\right)+g\left(t y_{1}+(1-t) y_{2}\right) \\
& \leq t f\left(x_{1}\right)+(1-t) f\left(x_{2}\right)+t g\left(y_{1}\right)+(1-t) g\left(y_{2}\right) \\
& =t \phi\left(z_{1}\right)+(1-t) \phi\left(z_{2}\right)
\end{aligned}
$$

Thus, $\phi(x, y)$ is convex.

\section{Competing interests}

The authors declare that they have no competing interests.

\section{Author details}

${ }^{1}$ Department of Engineering, Macquarie University, Balaclava Road, North Ryde, NSW, 2109, Australia. ${ }^{2}$ University of Technology, 15 Broadway, Ultimo, NSW, 2007, Australia. 
Received: 27 September 2014 Accepted: 17 March 2015 Published online: 09 April 2015

\section{References}

1. H Cao, V Leung, C Chow, H Chan, Enabling technologies for wireless body area networks: a survey and outlook. IEEE Commun. Mag. 47(12), 84-93 (2009)

2. M Patel, J Wang, Applications, challenges, and prospective in emerging body area networking technologies. IEEE Wireless Commun. 17(1), 80-88 (2010)

3. IEEE Standards Association, IEEE standard for local and metropolitan area networks - part 15.6: wireless body area networks. (IEEE, Piscataway, 2012). doi:10.1109/IEEESTD.2012.6161600

4. H Alemdar, C Ersoy, Wireless sensor networks for healthcare: a survey. Comput. Networks. 54(15), 2688-2710 (2010)

5. A Nosratinia, THunter, A Hedayat, Cooperative communication in wireless networks. IEEE Commun. Mag. 42, 68-73 (2004)

6. Y Liang, W Veeravalli, Resource allocation for wireless relay channels Asilomar Conference Signals Syst. Comput. 2, 1902-1906 (2004)

7. KT Phan, DHN Nguyen, T Le-Ngoc, Joint power allocation and relay selection in cooperative networks. Paper presented at IEEE global telecommunications conference, (Honolulu, HI, Dec. 2009)

8. Y Zhao, R Adve, TJ Lim, Improving amplify-and-forward relay networks: optimal power allocation versus selection. IEEE Trans. Wireless Commun. 6, 3114-3122 (2007)

9. A Bolis, D Smith, D Miniutti, L Libman, Y Tselishchev, Challenges in body area networks for healthcare: the MAC. IEEE Commun. Mag. 50(5), 100-106 (2012)

10. Y Chen, J Teo, JCY Lai, E Gunawan, KS Low, CB Soh, PB Rapajic, Cooperative communications in ultra-wideband wireless body area networks: channel modeling and system diversity analysis. IEEE J. Sel. Areas Commun. 27(1), 5-16 (2009)

11. XG Huang, HG Shan, XM (Sherman) Shen, On energy efficiency of cooperative communications in wireless body area networks. Paper presented at IEEE wireless communications and networking conference. (Cancun, Quintana Roo, March 2011)

12. LS Wang, C Goursaud, N Nikaein, L Cottatellucci, J Gorce, Cooperative scheduling for coexisting body area networks. IEEE Trans. Wireless Commun. 12(1), 123-133 (2013)

13. RD Errico, R Rosini, M Maman, A performance evaluation of cooperative schemes for on-body area networks based on measured time-variant channels. Paper presented at IEEE international conference communications, (Kyoto, June 2011)

14. P Ferrand, M Maman, C Goursaud, JM Gorce, L Ouvry, Performance evaluation of direct and cooperative transmissions in body area networks. Ann. Telecommunications. 66, 213-228 (2011)

15. J Ding, E Dutkiewicz, XJ Huang, GF Fang, Energy-efficient cooperative relay selection for UWB based body area networks, Paper presented at IEEE international conference on ultra-wideband, (Sydney, NSW, Sep. 2013)

16. G Ntouni, A Lioumpas, K Nikita, Reliable and energy efficient communications for wireless biomedical implant systems. IEEE J. Biomed. Health Informatics. PP(99), 1-8 (2014)

17. HC Keong, TSP See, MR Yuce, An ultra-wideband wireless body area network: evaluation in static and dynamic channel conditions. Sens. Actuators A: Phys. 180, 137-147 (2012)

18. A Fort, C Desset, J Ryckaert, P DeDoncker, L VanBiesen, P Wambacq, Characterization of the ultra wideband body area propagation channel. Paper presented at IEEE international conference on ultra-wideband (2005)

19. A Khaleghi, R Chavez-Santiago, I Balasingham, Ultra-wideband statistical propagation channel model for implant sensors in the human chest. IET Microwaves, Antennas and Propagation. 5(15), 1805-1812 (2011)

20. KY Yazdandoost, K Sayrafian-Pour, Channel model for body area network (ban). IEEE P802.15 Working Group for Wireless Personal Area Networks (WPANs), Tech. Rep. Document IEEE 802.15-08-0780-05-0006 (2009)
21. S Boyd, L Vandenberghe, Convex optimization. (Cambridge University Press, New York, 2004)

22. G Breed, A summary of FCC rules for ultra wideband communications. High Frequency Electron. 4, 42-44 (2005)

23. MR Yuce, K Ho Chee, C Moo Sung, Wideband communication for implantable and wearable systems. IEEE Microwave Theory Tech. $\mathbf{5 7}$ 2597-2604 (2009)

\section{Submit your manuscript to a SpringerOpen ${ }^{\mathcal{O}}$ journal and benefit from:}

- Convenient online submission

Rigorous peer review

- Immediate publication on acceptance

- Open access: articles freely available online

- High visibility within the field

- Retaining the copyright to your article

Submit your next manuscript at $>$ springeropen.com 